\title{
Tunable and processable shape memory composites based on degradable polymers
}

\author{
Xi Zhanga, b, c, Mike A. Geven ${ }^{d}$, Dirk Grijpma ${ }^{d}$, Ton Peijs ${ }^{b, c}{ }^{*}$ and Julien E. Gautrot ${ }^{a, b^{*}}$ \\ alnstitute of Bioengineering, ${ }^{b}$ School of Engineering and Materials Science and ${ }^{\mathrm{c}}$ Nanoforce \\ Technology Ltd. Queen Mary, University of London, Mile End Road, London, E1 4NS, UK. \\ dMIRA Institute for Biomedical Technology and Technical Medicine, Department of \\ Biomaterials Science and Technology, University of Twente, the Netherlands
}

* To whom correspondence should be addressed E-mail: j.gautrot@qmul.ac.uk and t.peijs@qmul.ac.uk

\begin{abstract}
Biodegradable shape memory polymers are attractive materials for the design of biomedical scaffolds as they allow deploying implants remotely with minimal intervention, whilst allowing degradation and tissue repair. However, shape memory properties are difficult to design from common degradable polymers, without chemical modifications. Here were developed readily tunable processable shape memory polymer composites (SMPCs) based on established degradable polymers in the biomedical field (poly(trimethylene carbonate) (PTMC) and poly(lactic acid) (PLA) fibres). These SMPCs rely on the glass-rubber transition of the PLA network to trigger shape recovery, whilst the elastic PTMC matrix optimises the full recovery of the scaffold to permanent shape. We demonstrate the performance of SMPCs can be readily designed by adjusting the loading and processing of the fibre network, or through the addition of plasticising poly(ethylene glycol) chains. Importantly, we demonstrate that the use of cut fibres allows the solution processing of SMPCs, which should enable the design of potentially degradable shape memory 3D scaffolds with complex shapes.
\end{abstract}

Key words: shape memory polymers, composites, electrospun fibres 


\section{Introduction}

Shape memory polymers (SMPs) area group of polymeric materials which are capable of recovering from a temporary shape to a memorized permanent shape when triggered by an external stimulus. Compared to conventional shape memory alloys (SMAs) and ceramics, shape memory polymers (SMPs) display interesting features for biomedical applications: they allow high recoverable strains, compared to shape memory alloys, they are relatively compliant (an advantage for contact with soft tissues) and have low densities and costs [1,2]. SMPs are particularly interesting for biomedical applications, for example for the design of implants that can be compressed and inserted into patients before deployment, via minimally invasive surgery [3]. SMPs were also designed to be used as smart suture materials for wound closure [4]. In addition to temperature, SMPs can be controlled via a number of external stimuli (e.g., light, moisture, electrical etc.) [5-8]. Polyurethanes (PU), polyesters, polyaramides and polyimides are among commonly reported SMPs [2, 9-11]. Their shape memory behaviour is based on the occurrence of two domains within the polymer network: flexible domains conferring elastic behaviour and recovery and hard domains conferring fixation and controlling the trigger of recovery [2], although examples of shape memory polymers relying on one single domain and high chain entanglement below a glass transition have also been reported [9, 12].Typically, shape recovery is triggered via athermally-induced transition to a rubbery glassy state or via the melting of crystallites within the hard domains of the SMP.

Despite excellent shape memory performance (high fixities and recoveries over a wide range of recoverable strains), SMPs are associated with low strength and moduli, which may prevent their application in scaffolds requiring more rigid and tougher mechanical properties, for example via reinforcement with nanofibres or platelets [13]. Thus reinforcements were added into SMPs (known as shape memory polymer composites), which not only improved the SMPs' mechanical properties and shape-fixing efficiency, but also enabled the introduction of electroactive, magneto-active, photo-active or moisture sensitive responsiveness [14-16]. 
Recently, shape memory polymer composites (SMPCs) based on the combination of two polymers displaying no shape memory properties have been developed. In these materials, an elastomer conferring shape recovery is combined with a fixing phase providing shape fixity. Luo and Mather reported the fabrication of polymer-polymer SMPCs via the integration of crystallisable poly(carprolactone) (PCL) electrospun fibres into an elastic silicone rubber matrix [17]. Shape fixation and recovery were achieved via the crystallization and melting of PCL fibres, respectively. Similarly, Zhang et al. [18] reported the fabrication of SMPCs by direct melt-blending of styrene-butadiene-styrene block copolymer (SBS) and PCL. Robertson et al. prepared SMPCs by electrospinning interwoven PCL and polyurethane elastomer fibres followed by compression into films [19]. However, despite interesting thermo-mechanical properties, these materials were not found to be fully degradable and cannot be readily processed into complex 3D shapes, limiting their applications as scaffold materials for tissue engineering.

Biocompatible and degradable SMPs have been developed using degradable multiblock copolymers that display excellent shape memory effect with an excellent level of tunability in recovery temperature and high recoverable strains, shape fixities and recoveries [4, 20-22]. However, each new material requires new approval from regulatory bodies in order to be applied in implants or scaffolds, limiting or considerably slowing their translation. Therefore the use of a combination of approved polymers not displaying shape memory properties for the design of SMPCs is attractive from an industrial point of view. Recently, Lawton et al. reported fully degradable SMPCs based on a polyanhydride elastomer and PU-PCL electrospun fibres, as the fixation phase, allowing the control of the reconfiguration and triggering of shape recovery [23]. However, these materials are limited to simple geometries that can be generated using electrospun fibres deposited on a substrate and subsequently impregnated within a polyanhydride matrix.

Here we report the preparation of one-way polymer-polymer SMPCs based on solution processable poly(lactic acid) (PLA) fibres and crosslinked poly(trimethylene carbonate) 
(PTMC) networks. Although the PLA fibres we used were manufactured via electrospinning (to achieve micron size fibres), these were cut into short fibres (though preserving high aspect ratios, $>100$ ) and resuspended in order to confer solution processability. PLA is a commercially-available, well-established degradable and biocompatible polymer with applications in the biomedical field, including tissue engineering [24]. Crosslinked PTMC networks, based on the curing of methacrylate functionalised PTMC macromers, were also shown to be fully biocompatible and degradable $[25,26]$. In addition, crosslinked PTMC can be structured via stereolithography in order to generate tissue engineering scaffolds for cartilage or bone regeneration [27]. In the polymer-polymer SMPCs we developed, the crosslinked PTMC matrix acts as the flexible domain that enables shape recovery whilst the PLA fibres act as hard domains providing shape fixity, allowing a triggered recovery at their glass transition. The large interfacial area and continuity of the networks formed by the high aspect ratio fibres and the matrix enables the efficient stress transfer that is induced during shape fixity and recovery [28]. We compare the performance of SMPCs generated from continuous and cut electrospun PLA fibres and show that moderate loading of fibres is sufficient to achieve excellent shape memory properties. In addition, the ease with which both phases (PTMC and PLA) can be engineered separately enables to control shape recovery and fixity as well as the triggering temperature. Finally, these short, cut fibre-based composites can be processed into complex shapes by traditional moulding techniques such as injection, extrusion or compression moulding, vastly broadening the scope of applications of SMPCs. 


\section{Experimental section}

\subsection{Materials}

Three-armed methacrylate-ended PTMC macromer with molecular weight of $5,000 \mathrm{~g} / \mathrm{mol}$, $10,000 \mathrm{~g} / \mathrm{mol}$ and $17,200 \mathrm{~g} / \mathrm{mol}$ were synthesized as previously reported [29]. PLA 2002D (Mw 200,000 , density $1.24 \mathrm{~g} / \mathrm{cm}^{3}$, tensile modulus $3.5 \mathrm{GPa}$ ) was purchased from NatureWorks. PEG (Mw 8,000), propylene carbonate and ethanol were purchased from Sigma-Aldrich. Chloroform, dichloromethane (DCM) and dimethylformamide (DMF) were obtained from Fisher Scientific. Irgacure TPO-L and Orasol Orange 247 were supplied by BASF. All polymers and reagents were used as received.

\subsection{Electrospinning and short fibre preparation}

For PLA electrospinning, PLA 2002D was dissolved in a mixture of chloroform and DMF (mass ratio 3/1) at a concentration of $9 \mathrm{wt} \%$. A syringe pump (Kent Genie) was used to continuously supply the PLA solution at $1.0 \mathrm{ml} / \mathrm{h}$ to a blunt spinning needle through a PTFE tube. For PLAPEG electrospinning, 10wt\% PEG was added to the PLA. The blends were dissolved in DCM at a total polymer concentration of $9 \mathrm{wt} \%$. The PLA-PEG solution was supplied to the spinning needle at $2.0 \mathrm{~mL} / \mathrm{h}$ using a syringe pump. The spinning distance was $15 \mathrm{~cm}$ (from needle to collector). A voltage of 20-25 kV (Glassman EQ) was applied to the needle to induce spinning. Random fibre mats were collected on a grounded aluminium foil sheet, and kept in vacuum desiccator for $48 \mathrm{hrs}$ to remove residual solvent after spinning.

Short PLA fibres were prepared as previously described [28]. Briefly, electrospun PLA fibres were mechanically stirred in toluene/petroleum ether media $\left(\mathrm{V}_{\text {toluene }} / \mathrm{V}_{\text {petroleum ether }} 80 / 20\right)$ at $1,500 \mathrm{rpm}$ for $24 \mathrm{hrs}$. Fibres with different length were obtained by mechanical stirring of asspun fibres in $V_{\text {toluene }} / V_{\text {petroleum ether }} 70 / 30$ at 1,500 rpm for 24 hrs. For simplicity of description, these two types of short fibres are named as L1 and L2, corresponding to 80/20 and 70/30 solvent ratios respectively, throughout our manuscript. The resulting fibres were recovered by 
filtering. Recovered fibres were dried in a fume hood overnight and then transferred into a vacuum desiccator for $48 \mathrm{hrs}$ to remove residual solvents.

\subsection{Fabrication of SMPCs}

Continuous fibre composites were fabricated by casting PTMC/propylene carbonate solutions onto the electrospun PLA fibre mats [28]. The PTMC concentration was $40 \mathrm{wt} \%$. Photo initiators Irgacure TPO-L and Orasol Orange 247 were added to the PTMC/propylene carbonate solution at the concentration of $5 \mathrm{wt} \%$ and $0.15 \mathrm{wt} \%$, respectively with regards to PTMC. The solution was slowly casted onto the electrospun fibre mats $(50 \times 20 \times 0.10 \mathrm{~mm}) . \mathrm{A}$ quartz plate was placed on top with gentle pressure applied to facilitate solution impregnation as well as to create a flat and smooth composite surface. The samples were then exposed to UV irradiation (Omnicure 1500) at $15 \mathrm{~mW} / \mathrm{cm}^{2}$ for $90 \mathrm{sec}$ to crosslink the matrix. The cured composite films were then extracted using propylene carbonate/ethanol to remove propylene carbonate. A daily refreshed propylene carbonate/ethanol media with decreasing propylene carbonate ratio (10 vol\% decrement daily) was used for extraction, which started from $\mathrm{V}_{\text {propylene }}$ carbonate $/ V_{\text {ethanol }} 50 / 50$ and finished until $0 / 100$. The fully extracted composites were left in a fume hood for 48 hrs to dry. Discontinuous PTMC/PLA fibre composites were prepared by first blending short fibres with $40 \mathrm{wt} \%$ PTMC/propylene carbonate solution and then casting the mixture into a $50 \times 20 \times 0.15 \mathrm{~mm}$ mould, which was then exposed to UV for crosslinking and extracted in the same way as continuous PTCM/PLA fibre composites. Four different fibre L1 loadings (5 wt \%, $10 \mathrm{wt} \%, 30 \mathrm{wt} \%$ and $50 \mathrm{wt} \%$ with respect to PTMC) were introduced into the PTMC composites. PTMC composites with $10 \mathrm{wt} \%$ and $30 \mathrm{wt} \%$ fibre L2 were prepared in the same way as fibre L1. For comparison, neat PTMC samples were produced by casting $40 \mathrm{wt} \%$ PTMC/propylene carbonate solution into a $50 \times 20 \times 0.15 \mathrm{~mm}$ mould, followed by the same UV crosslinking and extraction process. 


\subsection{Morphological characterization}

Scanning electron microscopy (SEM, FEI Inspect F) was used to characterize both fibres and composites. All samples were sputter coated with gold before analysis. The imaging was performed at $10 \mathrm{~mm}$ distance and a voltage of $10 \mathrm{kV}$. For the characterization of short fibres, a drop of fibre dispersion at the end of a mechanical stirring was transferred onto a cover slip and dried in a fume hood. For observing fibre-matrix integration, composites were immersed in liquid nitrogen and cold fractured. Software Image $\mathrm{J}$ was used to calculate the average diameters of the electrospun PLA fibres as well as the length of the discontinuous fibres.

\subsection{Thermomechanical analysis}

The thermomechanical properties of PTMC and PTMC/PLA fibre composites were characterized using dynamic mechanical analysis (DMA, TA instruments Q800). Tests were carried out in multi-frequency-strain mode at a pre-load of $0.05 \mathrm{~N}$, a frequency of $1 \mathrm{~Hz}$ and strain of $0.1 \%$, with $125 \%$ force track applied. Samples were cut into $20 \times 5 \mathrm{~mm}$ rectangular films (thickness 0.10-0.15 mm depending on sample type) and mounted into the DMA clamps. The temperature was first equilibrated at $-50{ }^{\circ} \mathrm{C}$ and then ramped to $100{ }^{\circ} \mathrm{C}$ at $3{ }^{\circ} \mathrm{C} / \mathrm{min}$. The storage modulus (E') and tensile loss tangent $(\tan (\delta))$ were monitored as a function of temperature. The glass transition temperature $T_{g}$ was determined by the temperature point when $\tan (\delta)$ reaches its maximum value.

Differential scanning calorimetry (DSC, PerkinEImer DSC 4000) was carried out to investigate the thermal properties of PLA and PLA-PEG fibres, PTMC matrix and fibre composites. A temperature scan was performed from $25{ }^{\circ} \mathrm{C}$ to $180{ }^{\circ} \mathrm{C}$ at a rate of $10{ }^{\circ} \mathrm{C} / \mathrm{min}$. The glass transition temperature $T_{g}$ was determined by the mid-point of the glass transition. The crystallinity of PLA fibres was calculated from the equation:

$$
X_{c}=\frac{\Delta H_{m}}{\Delta H_{\text {ref }}} \times \frac{1}{w} \times 100 \%
$$


where $\mathrm{X}_{c}$ is the crystallinity, $\Delta \mathrm{H}_{\mathrm{m}}$ is the experimental heat of fusion at the melting point as determined by DSC, $\Delta \mathrm{H}_{\text {ref }}$ is the theoretical heat of fusion of fully crystalline PLA (93 J/g) [30] and $w$ is weight fraction of PLA.

\subsection{Shape memory characterisation}

Shape memory tests were conducted in a DMA (TA instruments Q800) with samples cut into $20 \times 5 \mathrm{~mm}$ rectangular films (thickness $0.10-0.15 \mathrm{~mm}$ depending on sample type). Controlled force mode was used in the tests. First, a stress-strain test of each sample at $T_{g}+20^{\circ} \mathrm{C}\left(T_{g}\right.$ of PLA or PLA-PEG blend) was performed to determine the suitable static force to be used for the shape memory test. Samples were preloaded by $0.01 \mathrm{~N}$ and run at $0.1 \mathrm{~N} / \mathrm{min}$ force ramp until sample failure or reaching maximum equipment displacement. Then in each single cycle of the shape memory test, samples were preloaded by $0.01 \mathrm{~N}$ and equilibrated at $\mathrm{T}_{\mathrm{g}}+20^{\circ} \mathrm{C}$. They were then stretched to $40-50 \%$ strain at a force ramp of $0.1 \mathrm{~N} / \mathrm{min}$ (except for PTMC/50 wt $\%$ fibres L1, which was stretched to $20 \%$ strain). The final static force used here was determined by prior stress-strain test at $\mathrm{T}_{g}+20^{\circ} \mathrm{C}$. After holding the load at this temperature for 5 min, samples were cooled down to $\mathrm{T}_{\mathrm{g}}-20^{\circ} \mathrm{C}$ at $-10^{\circ} \mathrm{C} / \mathrm{min}$ while maintaining the static force. This static force was quickly released at $\mathrm{T}_{\mathrm{g}}-20^{\circ} \mathrm{C}$ and samples were allowed to relax for $5 \mathrm{~min}$. The temperature was finally raised back and equilibrated at $\mathrm{T}_{g}+20^{\circ} \mathrm{C}$, triggering sample shape recovery. Another three consecutive loading cycles were performed on each sample. For comparison, SME tests on neat PTMC were programmed in the same way as for the PTMCfibre composites at $\mathrm{T}_{g}+20{ }^{\circ} \mathrm{C}\left(\mathrm{T}_{g}\right.$ of PLA or PLA-PEG blend).

To quantify the shape memory properties of each sample, the strain fixity $\left(R_{f}\right)$ and strain recovery $\left(R_{r}\right)$ were calculated, according to the following equations:

$$
\mathrm{R}_{\mathrm{f}}=\frac{\varepsilon_{\mathrm{u}}(\mathrm{N})}{\varepsilon_{\mathrm{m}}}
$$

and 


$$
\mathrm{R}_{\mathrm{r}}(\mathrm{N})=\frac{\varepsilon_{\mathrm{m}}-\varepsilon_{\mathrm{p}}(\mathrm{N})}{\varepsilon_{\mathrm{m}}-\varepsilon_{\mathrm{p}}(\mathrm{N}-1)}
$$

where $\mathrm{N}$ is the number of cycles tested, $\varepsilon_{\mathrm{m}}$ is the maximum strain that the sample is stretched to, $\varepsilon_{u}$ is the strain of the sample after relaxing at $58^{\circ} \mathrm{C}\left(\mathrm{T}_{\mathrm{g}}-20^{\circ} \mathrm{C}\right)$ and $\varepsilon_{\mathrm{p}}$ is the strain of the sample after recovering at $98^{\circ} \mathrm{C} .100 \%$ of $R_{f}$ and $R_{r}$ means complete shape fixing and recovering, respectively.

\section{Results and Discussion}

\subsection{PTMC-impregnated PLA fibre mats display shape memory properties}

To generate PTMC/PLA composites, we first electrospun PLA fibre mats, to be impregnated with PTMC polymer solution. Characterization of the fibres using SEM demonstrated a continuous network of smooth fibres (see Figure 1a). The fibre diameter was found to vary from $0.4 \mu \mathrm{m}$ to $3 \mu \mathrm{m}$, with an average diameter of $1.2 \mu \mathrm{m}$. SEM images of cold-fractured PTMC/PLA fibre composites are shown in Figure 1b. By measuring the mass of the electrospun fibre mat before and after PTMC infiltration, the mass ratio of continuous PLA fibre in PTMC composites was estimated at $30.0 \pm 3.0 \%$. Compared with neat PTMC (see Figure S1a, in Supplementary material) the material's fracture surface is rougher with fibres protruding, while at the same time displaying excellent incorporation of the fibres within the matrix. The absence of significant fibre pull-out after cold-fracture further demonstrated the excellent interaction between the PLA fibres and the PTMC matrix. For continuous fibre composites, the interconnected space between the electrospun fibres is completely filled with PTMC matrix and no cavities are observed, suggesting excellent impregnation and wetting of the PTMC solution and resulting matrix. Together with the strong interfacial bonding between 
fibres and matrix, these SMPCs should display excellent stress transfer associated with an effective reinforcement and increase in mechanical properties.

PTMC displays a clear sharp glass transition near $-4^{\circ} \mathrm{C}$, particularly apparent in dynamic mechanical analysis (DMA) experiments, associated with a 3 order of magnitude drop in the storage modulus (Figure 1c). This is in good agreement with the thermal properties reported for PTMC and the soft mechanical properties expected from crosslinked PTMC networks in the rubbery state [31]. Similarly, PTMC/PLA composites display a first drop in storage modulus near $-4^{\circ} \mathrm{C}$, followed by a second drop at $78^{\circ} \mathrm{C}$, corresponding to the glass transition of PLA fibres. Both transitions are associated with a 40 -fold change in the mechanical properties of the composite. The magnitude of these changes and their occurrence on either side of room temperatures implied that PTMC/PLA composites should display shape memory properties, with the rubbery soft PTMC phases providing shape recovery and the switchable PLA fibre network conferring shape fixity. PTMC/PLA composites were also characterized by differential scanning calorimetry (DSC) to study the effect on crystallinity of PLA. DSC tests confirmed the occurrence of the second glass transition in PTMC/PLA composites at a temperature of $60^{\circ} \mathrm{C}$ (Figure S1b). Interestingly, the crystallinity of the PLA fibres increased from $3.4 \%$ to 37.1\% after incorporation into PTMC composites due to solvent induced crystallization [32], therefore further rigidifying the hard domains of the composite. Such effect may contribute to the fixation of the temporary shape of the samples (see discussion below). 

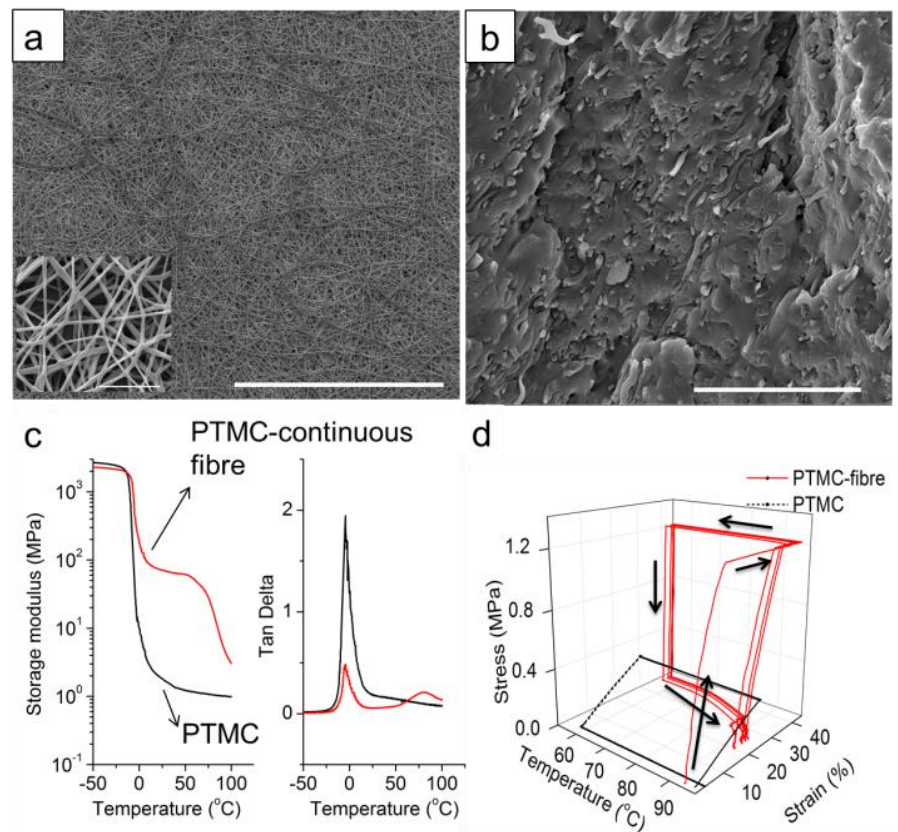

d
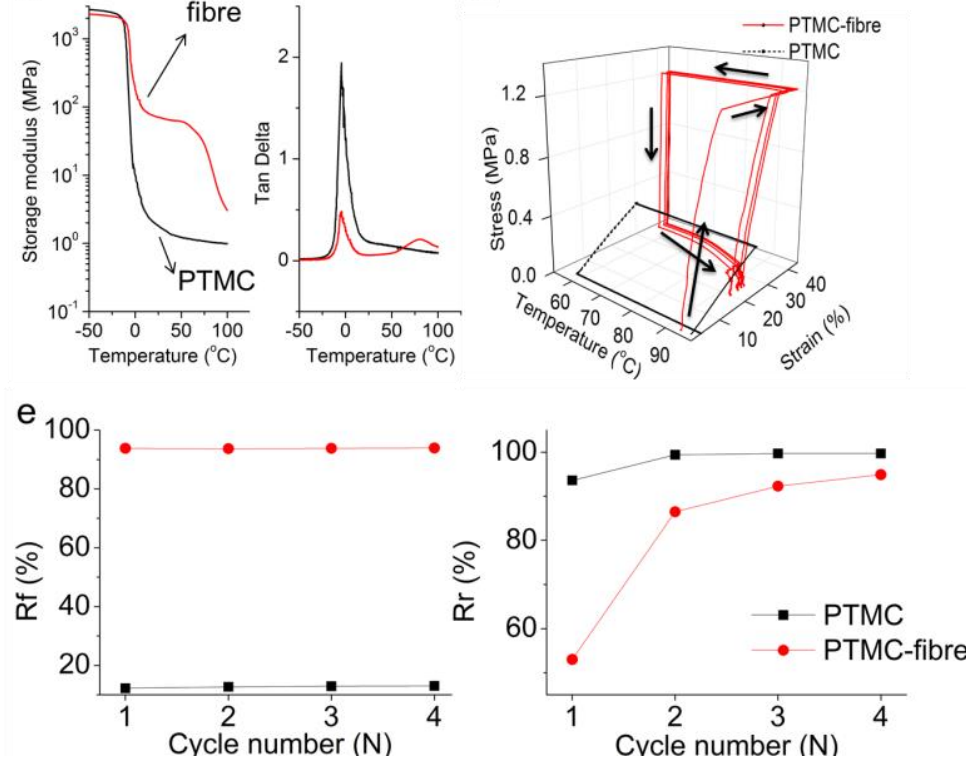

Figure 1. a) SEM images of electrospun PLA fibres (scale bar $400 \mu \mathrm{m}$ ), inset shows an electrospun PLA fibre mat at higher magnification (scale bar $20 \mu \mathrm{m}$ ). b) SEM images of coldfractured continuous PTMC/PLA fibre composites; c) Storage modulus and tan ( $\delta)$ of neat PTMC and continuous PTMC/PLA fibre composites as function of temperature, characterised by DMA. d) Representative temperature-stress-strain plots showing shape memory test cycles of neat PTMC (black dashed) and continuous PTMC/PLA fibre composites (red solid). e) Shape fixity ratio $\left(R_{f}\right)$ and shape recovery ratio $\left(R_{r}\right)$ against testing cycle number $(N)$ (measurements were carried out at $40 \%$ strain).

Tensile tests were carried out to determine the range of strains achievable for thermomechanical cycling experiments. Samples were loaded under tension at $98^{\circ} \mathrm{C}\left(\mathrm{T}_{\mathrm{g}}+20^{\circ} \mathrm{C}\right)$. Stress-strain traces and associated quantification of mechanical properties of the composites 
are presented in Figure $\mathbf{S 2}$ and Table S1 (see Supplementary material). The Young's modulus and tensile strength of PTMC increased from 1.69 to $16.6 \mathrm{MPa}$ and 0.58 to $1.97 \mathrm{MPa}$, respectively, after the introduction of the continuous PLA fibres. Failure strain is also improved more than three times from $51 \%$ to $176 \%$. These results are in good agreement with the increase in mechanical properties achieved by the incorporation of PLA fibres within the PTMC matrix as reported in our previous publication [28].

Representative temperature-stress-strain plots of a shape memory test cycle of PTMC and a PTMC/PLA fibre composite are presented in Figure 1d. The shape fixity and recovery ratios, $R_{f}$ and $R_{r}$, at different thermomechanical cycle numbers $(N)$ are presented in Figure 1eand Table S2 (Supplementary material). Continuous PTMC/PLA composites are seen to have excellent shape fixity with an average $R_{f}$ of $94 \%$. This may be linked to the relatively high crystallinity observed for PLA fibres within SMPCs, and associated strengthening of the hard network, expected to play an important role in defining the shape fixation properties of our composites, balancing elasticity stored in the PTMC matrix. In its first test cycle, a permanent strain of $20 \%$ was not recovered, presumably due to relaxation of PLA chains during loading at $98^{\circ} \mathrm{C}$, as well as processing history [11]. PLA chains are typically relatively oriented along the electrospun nanofibres, due to the extensional flow and high associated shear forces occurring during the solution jet of the electrospinning process [33]. Hence molecular relaxation occurring during initial thermomechanical cycles above the $T_{g}$ of PLA fibres is expected to account for some of the initial loss in shape recovery. However, upon subsequent cycling, strain recovery ratios $\mathrm{R}_{\mathrm{r}}$ increased up to $95 \%$. In addition, compared to SMPCs based on discrete phases [18], a continuous network of hard domains results in improved stress transfer and associated shape fixity ratio $R_{f}$. SEM of neat PTMC and PTMC/PLA fibre composites after thermomechanical cycling experiments confirmed that no significant morphological defects were observed within SMPCs after repeated shape memory cycles (Figure S5). These images confirmed the strong interfacial bonding between PLA fibres and the PTMC matrix, with no apparent fibre pull-out, even after repeated shape memory cycling. 


\subsection{Impact of the PTMC matrix on SMPCs performance}

The impact of the mechanical properties of the PTMC elastomeric phase on the shape memory properties of SMPCs was investigated next. In order to control the mechanical properties of the PTMC matrix, the molecular weight of the PTMC macromers was altered (from Mw5,000 to $17,200 \mathrm{~g} / \mathrm{mol}$ ). Corresponding composites were prepared using PLA fibre mats similar to PTMC $10,000 \mathrm{~g} / \mathrm{mol}$-based samples. Composites generated using PTMC $5,000 \mathrm{~g} / \mathrm{mol}$ matrix displayed excellent integration and wet-out of PLA fibres and no apparent fibre pull-out was observed in cold fractured samples. However, the integration of the fibre mats within the matrix of PTMC $17,200 \mathrm{~g} / \mathrm{mol}$ was found to be poorer, with apparent voids between fibres and a matrix-rich layer remaining at the surface of the composite suggesting poor impregnation due to the higher polymer viscosity (Figure 2). The $T_{g}$ of the PTMC phase of the composites shifted to $-1.9^{\circ} \mathrm{C}$ and $-8.8^{\circ} \mathrm{C}$, for PTMC 5,000 and $17,200 \mathrm{~g} / \mathrm{mol}$ respectively. Stress-strain tests $\left(\right.$ at $98^{\circ} \mathrm{C}$ )indicated that neat PTMC 5,000 has a higher modulus with a lower failure strain while neat PTMC 17,200 is more compliant with a considerably higher failure strain, compared to PTMC 10,000 (see Table S1), consistently with the expected change in crosslink density for these different PTMC matrices. Neat PTMC 5,000 did not display any shape memory properties, whereas some level of fixity was observed for neat PTMC 17,200, as a result of viscoelastic creep (see Table S2). In turn, due to the increased stiffness for PTMC 5,000, composites based on this matrix displayed poor shape fixities, as the PLA fibre network was not sufficiently strong to fix the temporary shape of the sample and resist the stress stored within the PTMC matrix. However, the recovery ratio of PTMC 5,000 composites was improved, as expected. In agreement with this observation, composites based on PTMC 17,200 displayed increased fixities but lower recoveries (see Table S2). 

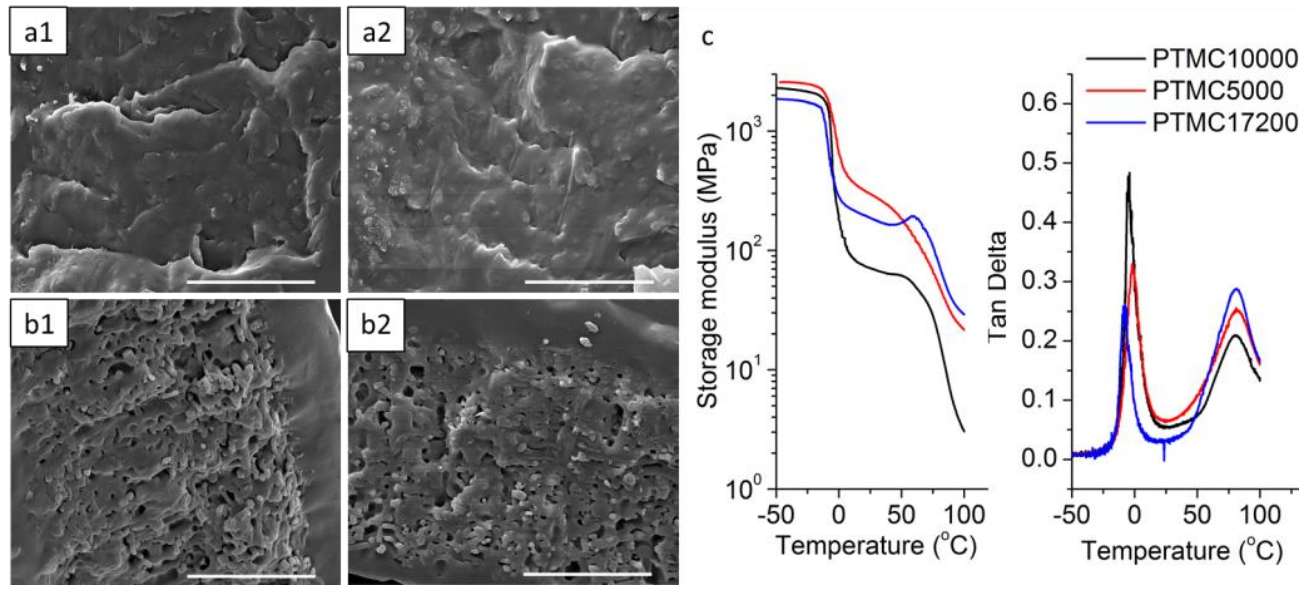

Figure 2. SEM images of PTMC 5000/PLA fibre composites before (a1) and after (a2) shape memory tests; SEM images of PTMC 17200/PLA fibre composites before (b1) and after (b2) shape memory tests. The scale bar for $a$ and $b$ is $20 \mu \mathrm{m}$. (c) Storage modulus and tan ( $\delta$ ) of PTMC/PLA fibre composites as function of temperature tested by DMA (black-PTMC 10,000, red-PTMC 5,000, blue-PTMC 17,200)

\subsection{SMPCs based on solution-processable cut eletrospun PLA fibres}

A major disadvantage of SMPCs based on electrospun fibre mats is the limited range of scaffold geometries and design that can be manufactured. In order to tackle this issue, we investigated next whether solution-processable composites based on a PTMC matrix and cut PLA electrospun fibres could be designed and display shape memory properties. Continuous electrospun PLA fibres were successfully cut into short fibres by stirring in toluene/petroleum ether 80/20 (named as fibresL1; see representative SEM image in Figure 3a). Fibre L1 display an average length of $220 \pm 110 \mu \mathrm{m}$ and their length distribution can be found in Figure S3 (Supporting Information). Fibre L1 were suspended in PTMC/propylene carbonate solutions at different loadings $(5,10,30$ and 50 wt\% with respect to PTMC) and crosslinked to form short fibre composites. Similar to impregnated continuous fibre mat-based composites, the short fibre composites displayed excellent integration of the fibres within the PTMC matrix (see

Figure $\mathbf{3 b}$ and Figure S4), essential for the effective reinforcement of the PTMC-based 
composite [28]. Another important parameter in the design of short fibre composites is the critical fibre length, which is required to be much greater than the critical length, for effective reinforcement. In our previous work, we had calculated that the critical length $L_{c}$ of such short PLA fibres in PTMC was $21 \mu \mathrm{m}$ [28], an order of magnitude below the average length of fibre $L 1$, indicating that these short fibres, with lengths of $10 x L_{c}$, should be sufficient long to efficiently transfer stresses induced during shape fixity [34]. DSC and DMA confirmed the similar thermomechanical properties of the short fibre composites, compared to those based on impregnated mats (Figure S1and Figure 3c), with glass transitions at 60 and $78{ }^{\circ} \mathrm{C}$, based on DSC and DMA, respectively. Hence thermomechanical cycling was carried out in identical conditions to characterise the shape memory properties of the resulting short fibre L1 composites. Tensile tests (Figure S2 and Table S1) confirmed the strengthening effect of fibres L1, with increasing Young's moduli at increasing loading contents, although not to the same extent as for continuous fibre mats. Such weaker mechanical properties may be the result of a lower level of fibre entanglement in cut fibre composites, compared to impregnated electrospun fibre mats [35]. However, the range of recoverable strains achieved with composites incorporating the highest fibre loadings (50 wt\%) was reduced to $85 \%$. The decrease in failure stress and strain measured for such composites can be explained by the low yield strain of PLA and stress concentrations arising from fibre aggregates [28, 36]. 

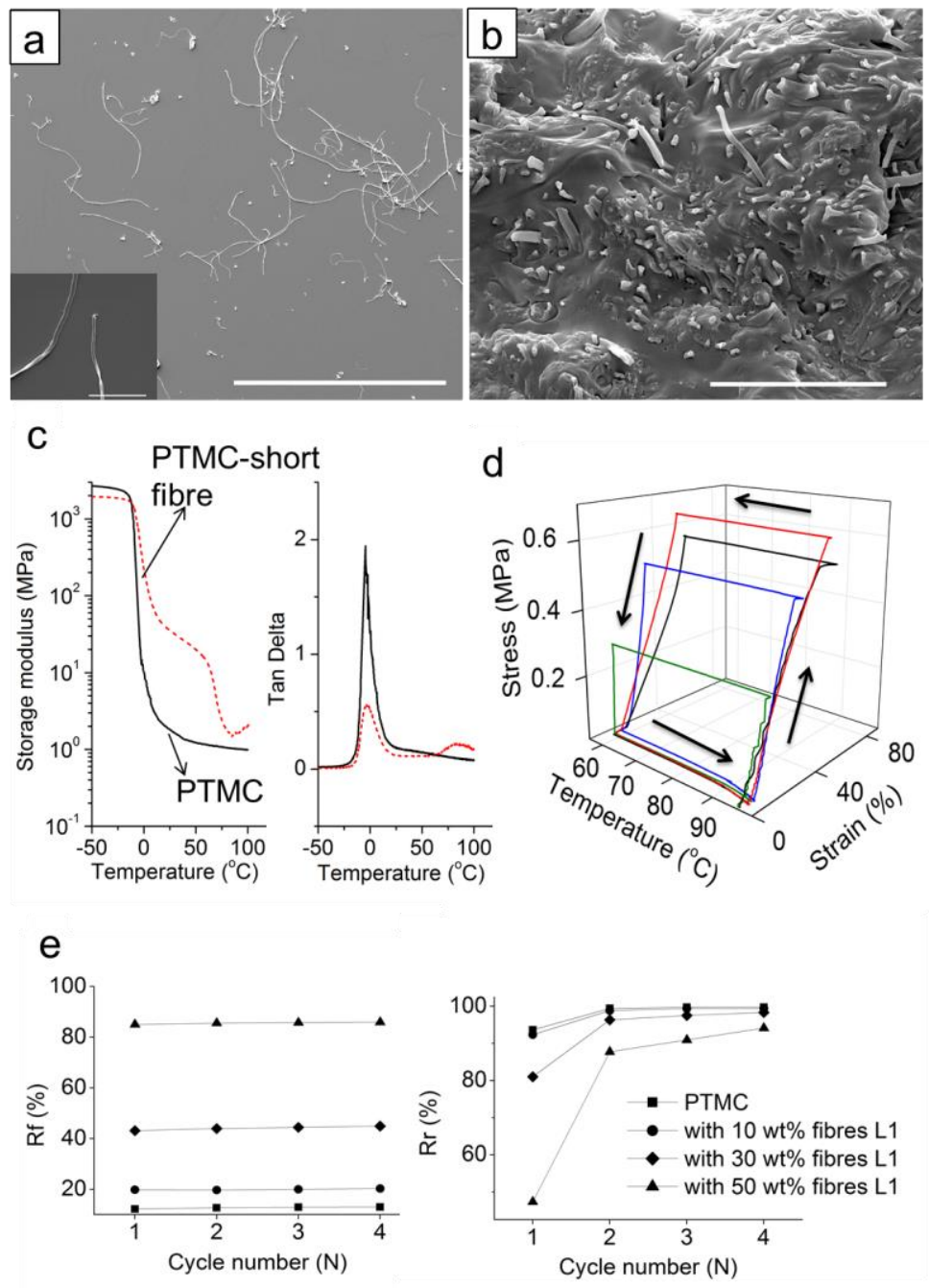

Figure 3. a) SEM images of electrospun PLA fibre L1 (scale bar $400 \mu \mathrm{m}$ ), inset shows PLA fibre L1 at higher magnification (scale bar $20 \mu \mathrm{m}$ ).b) SEM images of cold-fractured PTMC/30 wt\% fibre L1 composites. c) Storage modulus and tan (ס) of PTMC and PTMC/30 wt\% fibre L1 composites as function of temperature tested by DMA. d)Representative temperaturestress-strain plots showing shape memory test cycle of PTMC with different fibre L1 loadings (black: 5 wt $\%$, red: 10 wt $\%$, blue: 30 wt $\%$, green: $50 w t \%)$. e) Shape fixity ratio $\left(R_{f}\right)$ and shape recovery ratio $\left(R_{r}\right)$ against test cycle number $(N)$ (measurements were carried out at $50 \%$ strain for PTMC/5 wt\% and PTMC/10 wt\% fibre L1, 40\% strain for PTMC/30\% fibre L1, 20\% strain for PTMC/50wt\% fibre L1 respectively). 
The shape memory properties of these short fibre composites were investigated next. Thermomechanical cycling indicated lower shape fixity ratios $R_{f}$, compared to continuous fibre composites, except for composites loaded with 50 wt\% fibres, which displayed comparable fixities and recovery profiles to those observed for continuous fibre mats. The better fixities and recoveries observed for PTMC composites based on continuous PLA fibre mats is likely the result of an additional strengthening effect originating from the entangled fibrous network in such mats. Short fibre composites, on the other hand, do not exhibit such a network, particularly at low fibre loadings, which reduces the ability of the composite system to resist stresses originating from the PTMC matrix in order to maintain the temporary shape.

To investigate the impact of the continuous fibre network on composites' shape memory behaviour, short fibres with longer average lengths were prepared by mechanical stirring asspun fibres in toluene/petroleum ether $70 / 30$, resulting in slightly longer fibre L2, with an average length at $270 \pm 140 \mu \mathrm{m}$, i.e. $13 x \mathrm{~L}_{c}$ (see Figure S3). By decreasing the toluene fraction during mechanical stirring, the average fibre length was increased due to the lower swelling of PLA [28]. Two different loadings of fibresL2 (10 and $30 \mathrm{wt} \%$ ) were incorporated into the PTMC composites. Composites containing 50 wt\% fibre L2 were found to be difficult to prepare due to associated higher fibre entanglement and poorer dispersion within the PTMC solution. Incorporation of L2 fibre within the matrix remained excellent at 10 and 30 wt\% (Figure S4), with composites based on fibre L2 exhibiting similar thermomechanical properties, compared to systems based on fibre L1 (Figures S1and Table S1), in agreement with the average lengths of both fibres being much longer than the critical length for matrix reinforcement (i.e. $L 1=10 x L_{c}$ and $\left.L 2=13 x L_{c}\right)[34]$.

Characterisation of the shape memory properties of composites generated using fibre L2 indicated an increase in shape fixity, compared to composites based on fibre $L 1$ (at $10 \mathrm{wt} \%, \mathrm{R}_{\mathrm{f}}$ increases from $20 \%$ to $61 \%$; at $30 \mathrm{wt} \% \mathrm{R}_{\mathrm{f}}$ increases from $45 \%$ to $64 \%$ ). This is thought to result from the higher aspect ratio of L2 fibre and the associated increased entanglement network contribution for these fibres [37]. Composites with fibre L2 displayed a more 
pronounced training effect, but eventually excellent shape recovery (near 95\%). To further support the importance of fibre entanglements on the performance of SMPCs, a suspension of fibre L1was deposited into moulds $(50 \times 20 \times 1 \mathrm{~mm})$ and rectangular highly entangled fibre mats were formed after evaporation of the solvent(see SEM images, Figure 4b). A PTMC macromer/propylene carbonate solution was subsequently cast onto these mats to generate impregnated fibre mats from cut fibre L1 (with a final fibre loading of 30 wt\%). Compared to composites based on $30 \mathrm{wt} \%$ fibre L1 suspensions, stress-strain tests did not indicate any significant change in mechanical properties (Table S1). However, the shape fixity ratio $R_{f}$ increased from $45 \%$ to $75 \%$, while composites retained excellent shape recoveries $\left(R_{r}\right.$ of $97 \%$ in fourth test cycle). Overall, our results demonstrate that increasing the interconnection of the fibrous network, through an increased fibre loading, aspect ratio and entanglements, can lead to improved shape memory properties of the composites, in particular fixity ratios.
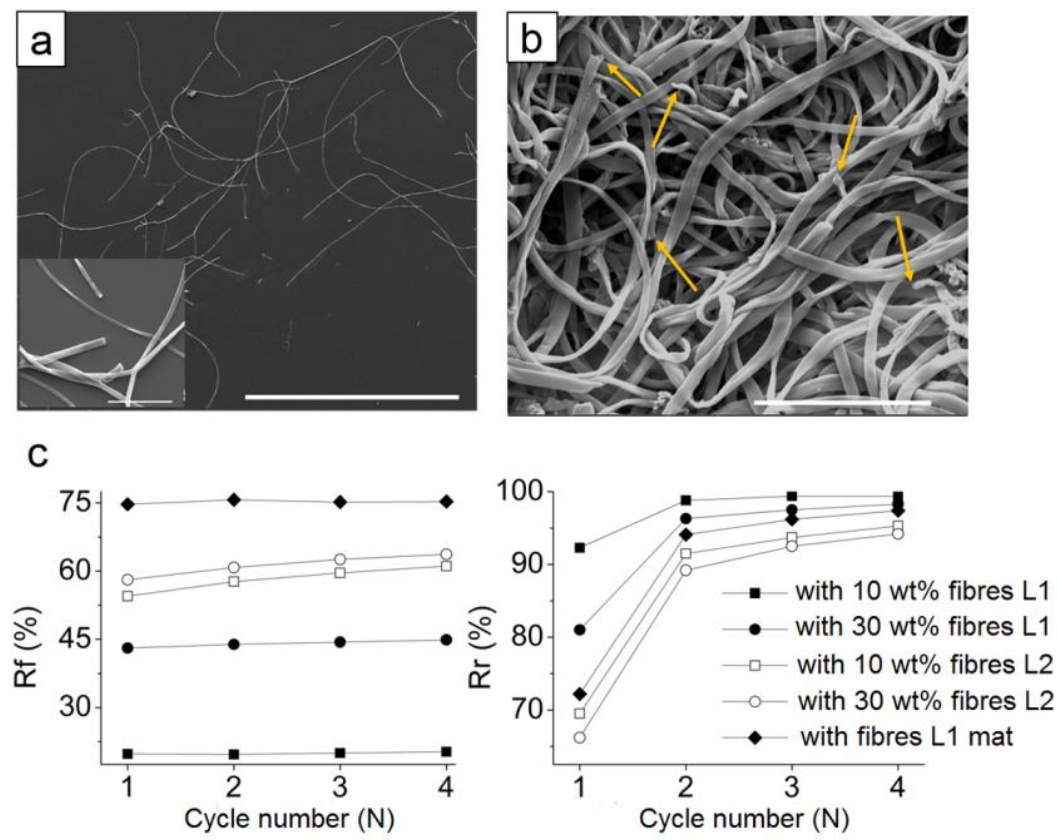

Figure 4. a) SEM images of fibre L2 (scale bar $400 \mu \mathrm{m}$ ), inset shows fibre $\mathrm{L} 2$ at higher magnification (scale bar $20 \mu \mathrm{m}$ ).b) SEM images of suspension-deposited fibre L1 mat (scale bar $20 \mu \mathrm{m}$, yellow arrows indicating fibres' ends). c) Shape fixity ratio $\left(\mathrm{R}_{\mathrm{f}}\right)$ and shape recovery 
ratio $\left(R_{r}\right)$ as a function of test cycle number $(N)$ (all measurements were carried out at $40 \%$ strain).

Images of shape memory behaviour obtained for SMPCs are presented in Figure 5. Rectangular samples (permanent shape: $20 \times 3 \times 0.12 \mathrm{~mm}$ ) were cut out from the continuous fibre composite films and placed on a hot plate heated at $100^{\circ} \mathrm{C}$. The sample was deformed and the temporary shape quickly quenched in cold water. The sample retained this temporary shape after releasing it, but quickly recovered when heated to $100^{\circ} \mathrm{C}\left(\mathrm{T}_{\mathrm{g}}+20^{\circ} \mathrm{C}\right.$; full recovery within $15 \mathrm{sec}$ ). To demonstrate the processability of cut fibres composites, a spring-shaped (permanent shape) sample was produced using a spiral tube mould. The temporary spiral shape was obtained by compressing the spring-like sample in a water bath at $90{ }^{\circ} \mathrm{C}$ followed by quenching in cold water. Shape recovery was achieved within $3 \mathrm{sec}$ at $90{ }^{\circ} \mathrm{C}$. Videos showing these recovery processes can be found in the Supplementary material (Video S1 and S2).

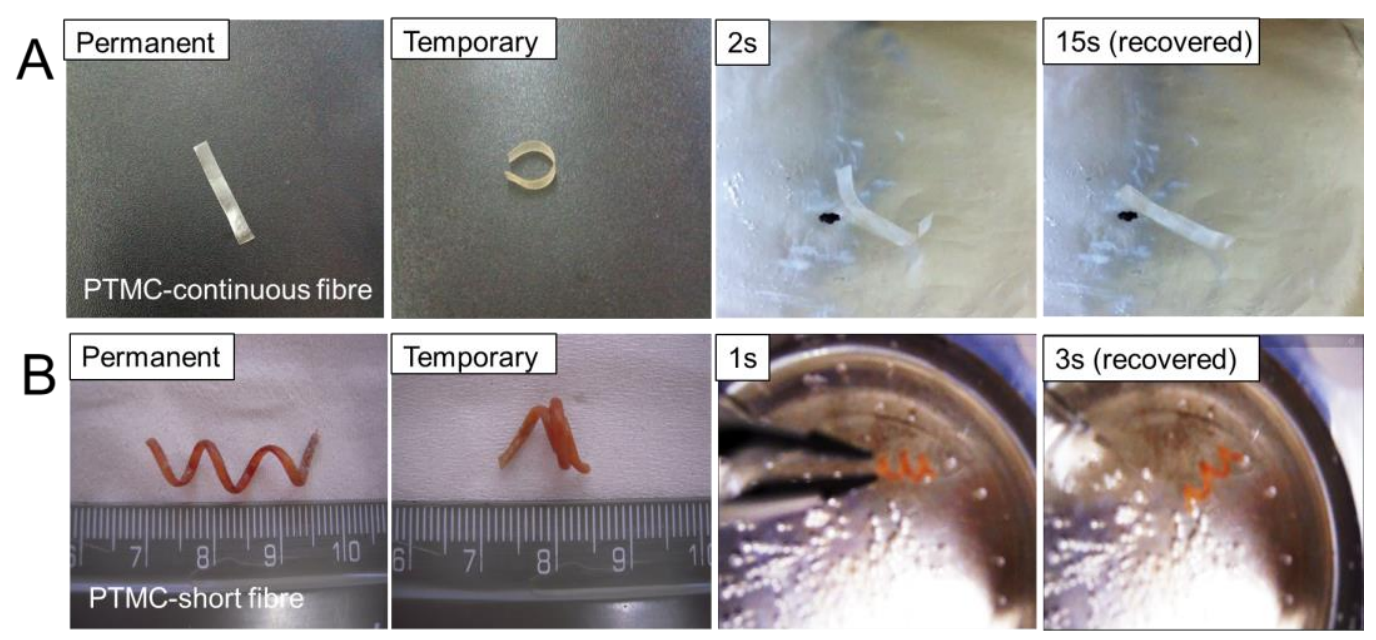

Figure 5. Photographs demonstrating PTMC/PLA fibres composite shape memory behaviour: (A) Rectangular continuous fibre composite sample was bend around a glass rod at $100{ }^{\circ} \mathrm{C}$ and then quenched. The shape recovery was triggered by heating to $100{ }^{\circ} \mathrm{C}$; $(\mathrm{B})$ a springshaped sample of short fibre composites (50 wt\% fibre L1) was compressed in a water bath 
$\left(90{ }^{\circ} \mathrm{C}\right)$ and quenched in cold water. By heating the sample back to $90{ }^{\circ} \mathrm{C}$, shape recovery was achieved.

\subsection{Modulation of the recovery temperature}

In order to control the recovery temperature at which the shape memory process is triggered in SMPCs, PEG was blended into PLA to act as a plasticiser and regulate the glass transition of the fibre network. PTMC/PLA-PEG composites were prepared and tested in the same way as PTMC/PLA fibre mat composites. SEM images of electrospun PLA-PEG fibres and cold fracture surfaces of these composites are shown in Figure S6. PLA-PEG fibres are continuous and defect-free, with an average diameter of $1.15 \pm 0.64 \mu \mathrm{m}$, with some very thin fibres present (150 nm). In addition, PLA-PEG fibres were found to integrate well to the PTMC matrix, as in PLA fibre composites. Blending of PLA reduced the $T_{g}$ of the electrospun blend fibres from 60 ${ }^{\circ} \mathrm{C}$ to $46{ }^{\circ} \mathrm{C}$ (with $10 \mathrm{wt} \% \mathrm{PEG}$ ), confirming its expected plasticising effect (see Figure S6). The crystallinity of the PLA-PEG fibres was found to increase compared to pristine PLA fibres (from $3.4 \%$ to $14.8 \%$ ), in agreement with previous reports [38]. After impregnation by the PTMC matrix, the crystallinity of the fibres increased to $41.6 \%$, whilst their $\mathrm{T}_{g}$ slightly increased to $50^{\circ} \mathrm{C}$. Such slight increase in $T_{g}$ is believed to be caused by the diffusion of PEG molecules during the impregnation process (prior to removal of propylene carbonate). Similarly, the glass transitions determined by DMA shifted from $-4{ }^{\circ} \mathrm{C}$ to $-19{ }^{\circ} \mathrm{C}$ and $78{ }^{\circ} \mathrm{C}$ to $68{ }^{\circ} \mathrm{C}$ (see Figure 6), further confirming that PEG is present and plasticises both the PTMC matrix phase and the fibre network. Thus the trigger temperature for characterisation of shape memory properties was set at $68 \pm 20^{\circ} \mathrm{C}$. Stress-strain tests at $88{ }^{\circ} \mathrm{C}$ indicated mechanical properties of PTMC/PLA-PEG composites comparable to PTMC/PLA composites, with a Young's modulus of $12.02 \pm 0.50 \mathrm{MPa}$, a tensile strength of $1.48 \pm 0.03 \mathrm{MPa}$ and yield strains of $191 \pm 10 \%$. The shape fixity $R_{f}$ and shape recovery ratios $R_{r}$ measured at different test cycles $(N)$ are presented

in Figure 6. PTMC/PLA-PEG fibre composites showed excellent shape fixities (averaging 97\%), and a comparable training effect to that observed for PTMC/PLA composites, although recoveries remained slightly below $90 \%$. Overall, our results demonstrate the feasibility of 
tuning the shape recovery temperature of SMPCs via the simple introduction of a plasticiser reducing the glass transition of the rigid fibre network.
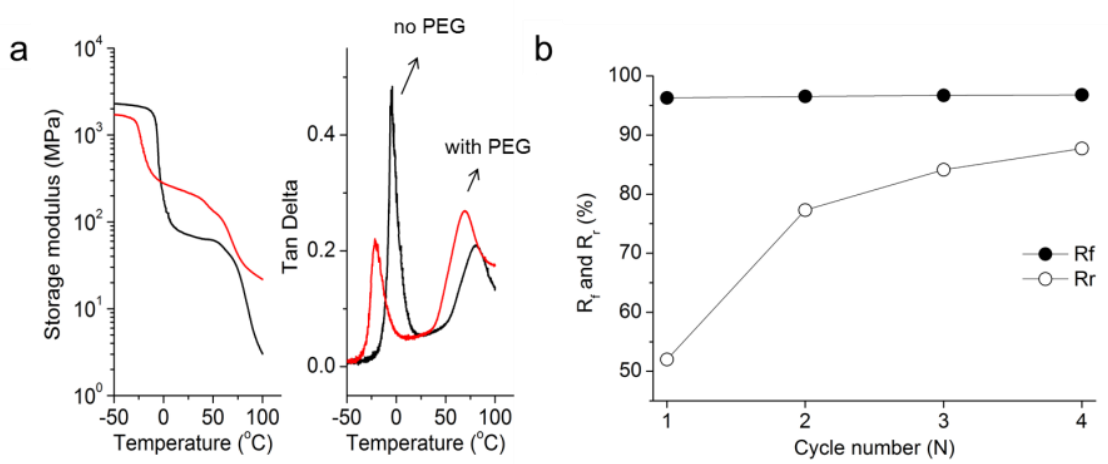

Figure 6. (a) Storage modulus and tan Delta of PTMC/PLA fibre composites as a function of temperature (with, red lines, and without PEG, dark lines). (b) Shape fixity ratio $\left(R_{f}\right)$ and shape recovery ratio $\left(R_{r}\right)$ at different test cycles $(N)$ (measurements were carried out at $40 \%$ strain).

Finally, PTMC/PLA-PEG composites were characterized by SEM and DMA after shape memory thermomechanical cycling. Fibres remained well integrated within the PTMC matrix (see Figure S7), confirming the integrity of the fibrous network after shape memory cycling. The storage modulus increased slightly after thermomechanical cycling, presumably a result of strain hardening and orientation during these tests. The position of the tan $(\delta)$ peaks of these composites remained unchanged, confirming the stability of the plasticizing effect (see Figure S7, Supplementary material).

\section{Conclusions}

One-way SMPCs based on biocompatible and biodegradable polymers were successfully developed by incorporating both continuous and cut electrospun PLA fibres into a crosslinked 
PTMC matrix. These fibres not only act as reinforcements, but also as a 'switching phase', whilst elastic recovery is conferred by the PTMC matrix. The large interfacial area and strong bonding between the PTMC matrix and electrospun PLA fibres underlie a highly efficient stress transfer mechanism accounting for the excellent shape memory properties measured. With the integration of $50 \mathrm{wt} \%$ short PLA fibres rather than continuous electrospun PLA fibre mats, processability of SMPCs was enabled, without compromising significantly the material's shape memory properties $\left(R_{f}=94 \%, R_{r}=95 \%\right.$ for continuous fibre mats and $R_{f}=86 \%, R_{r}=94 \%$ for 50 wt\% cut short fibres). We also demonstrated that the shape memory properties of SMPCs can be readily modulated by the design of the rigid fibrous network (degree of entanglement, plasticizing and control of the glass transition), as well as the thermomechanical properties of the matrix (mechanical properties). This is particularly interesting as this means that shape memory performance and triggering temperature can be readily adjusted in SMPCs without requiring further chemical modifications of their constitutive polymers and allows the design of such materials with off-the-shelf well-characterised, affordable and scalable polymers, already approved by regulatory bodies. In addition, the ability to solution process SMPCs based on cut short fibres should make these materials interesting candidates for the development of 3D printed scaffolds, for the flexible design of smart medical devices. However, important aspects should be further investigated to enable the use of SMPCs for the design of implantable scaffolds. Their biocompatibility and degradation behaviour should be studied in physiological conditions and in vivo. In addition, further modulation of the shape recovery temperature is required to enable actuation and deployment of scaffolds closer to body temperature. Plasticisers (biomedical grade) that are better compatible with PLA or other polyester fibres will help further reducing the glass transition temperature of the corresponding composites. 


\section{Acknowledgement}

The authors acknowledge the funding provided by NSFC-DG-RTD Joint Scheme (Project No. 51361130034) and the RAPIDOSproject under the European Union's 7th Framework Programme (Project No.604517).

\section{Supplementary material}

A video showing the shape recovery of PTMC/fibre composites can be found in the Supplementary material.

\section{Reference}

[1] Xie T. Recent advances in polymer shape memory. Polymer. 2011;52:4985-5000.

[2] Lendlein A, Kelch S. Shape-memory polymers. Angewandte Chemie International Edition. 2002;41:2034-57.

[3] Hampikian JM, Heaton BC, Tong FC, Zhang Z, Wong CP. Mechanical and radiographic properties of a shape memory polymer composite for intracranial aneurysm coils. Materials Science and Engineering: C. 2006;26:1373-9.

[4] Lendlein A, Langer R. Biodegradable, elastic shape-memory polymers for potential biomedical applications. Science. 2002;296:1673-6.

[5] Lendlein A, Jiang H, O. J, Langer R. Light-induced shape-memory polymers. Nature. 2005;434:87982.

[6] Huang WM, Yang B, An L, Li C, Chan YS. Water-driven programmable polyurethane shape memory polymer: Demonstration and mechanism. Applied Physics Letters. 2005;86:114105.

[7] Leng JS, Lan X, Liu YJ, Du SY, Huang WM, Liu N, Phee SJ, Yuan Q. Electrical conductivity of thermoresponsive shape-memory polymer with embedded micron sized Ni powder chains. Applied Physics Letters. 2008;92:014104.

[8] Song S, Feng J, Wu P. A New Strategy to Prepare Polymer-based Shape Memory Elastomers. Macromolecular rapid communications. 2011.

[9] Gautrot JE, Zhu XX. Shape Memory Polymers Based on Naturally-Occurring Bile Acids. Macromolecules. 2009;42:7324-31.

[10] Rabani G, Luftmann H, Kraft A. Synthesis and characterization of two shape-memory polymers containing short aramid hard segments and poly( $\varepsilon$-caprolactone) soft segments. Polymer. 2006;47:4251-60.

[11] Xiao X, Qiu X, Kong D, Zhang W, Liu Y, Leng J. Optically transparent high temperature shape memory polymers. Soft Matter. 2016;12:2894-900.

[12] Thérien-Aubin H, Gautrot JE, Shao Y, Zhang J, Zhu XX. Shape memory properties of main chain bile acids polymers. Polymer. 2010;51:22-5.

[13] Meng H, Li G. A review of stimuli-responsive shape memory polymer composites. Polymer. 2013;54:2199-221. 
[14] Luo X, Mather PT. Conductive shape memory nanocomposites for high speed electrical actuation. Soft Matter. 2010;6:2146.

[15] Luo X, Mather PT. Triple-Shape Polymeric Composites (TSPCs). Advanced Functional Materials. 2010;20:2649-56.

[16] Koerner H, Price G, Pearce NA, Alexander M, Vaia RA. Remotely actuated polymer nanocomposites--stress-recovery of carbon-nanotube-filled thermoplastic elastomers. Nature materials. 2004;3:115-20.

[17] Luo X, Mather PT. Preparation and Characterization of Shape Memory Elastomeric Composites. Macromolecules. 2009;42:7251-3.

[18] Zhang H, Wang H, Zhong W, Du Q. A novel type of shape memory polymer blend and the shape memory mechanism. Polymer. 2009;50:1596-601.

[19] Robertson JM, Birjandi Nejad H, Mather PT. Dual-Spun Shape Memory Elastomeric Composites. ACS Macro Letters. 2015;4:436-40.

[20] Xue L, Dai S, Li Z. Biodegradable shape-memory block co-polymers for fast self-expandable stents. Biomaterials. 2010;31:8132-40.

[21] Koji N, Yuichi U, Tatsuro O, Yuichi O. Biodegradable shape-memory polymers exhibiting sharp thermal transitions and controlled drug rlease. Biomacromolecules. 2009;10:1789-94.

[22] Rochette JM, Ashby VS. Photoresponsive Polyesters for Tailorable Shape Memory Biomaterials. Macromolecules. 2013;46:2134-40.

[23] Lawton MI, Tillman KR, Mohammed HS, Kuang W, Shipp DA, Mather PT. Anhydride-Based Reconfigurable Shape Memory Elastomers. ACS Macro Letters. 2016;5:203-7.

[24] Ulery BD, Nair LS, Laurencin CT. Biomedical applications of biodegradable polymers. Journal of Polymer Science Part B: Polymer Physics. 2011;49:832-64.

[25] Rongen JJ, Bochove B van., Hannink GJ, Grijpma DW, Buma P. Degradation behaviour of, and tissue response to photo-crosslinked poly(trimethylene carbonate) networks. Journal of Biomedical Materials Research Part A. 2016;104:2823-2832.

[26] Bat E, Kooten TG van., Feijen J, Grijpma DW. Resorbable elastomeric networks prepared by photocrosslinking of high-molecular-weight poly(trimethylene carbonate) with photoinitiators and poly(trimethylene carbonate) macromers as crosslinking aids. Acta Biomaterialia. 2011;7:1939-1948.

[27] Schuller-Ravoo S, Teixeira SM, Feijen J, Grijpma DW, Poot AA. Flexible and elastic scaffolds for cartilage tissue engineering prepared by stereolithography using poly(trimethylene carbonate)-based resins. Macromolecular bioscience. 2013;13:1711-9.

[28] Zhang X, Geven MA, Grijpma DW, Gautrot JE, Peijs T. Polymer-polymer composites for the design of strong and tough degradable biomaterials. Materials Today Communications. 2016;8:53-63.

[29] Geven MA, Varjas V, Kamer L, Wang X, Peng J, Eglin D, Grijpma DW. Fabrication of patient specific composite orbital floor implants by stereolithography. Polymers for Advanced Technologies. 2015;26:1433-8.

[30] Mathew AP, Oksman K, Sain M. The effect of morphology and chemical characteristics of cellulose reinforcements on the crystallinity of polylactic acid. Journal of Applied Polymer Science. 2006;101:300-10.

[31] Schuller-Ravoo S, Feijen J, Grijpma DW. Flexible, elastic and tear-resistant networks prepared by photo-crosslinking poly(trimethylene carbonate) macromers. Acta biomaterialia. 2012;8:3576-85.

[32] Sato S, Gondo D, Wada T, Kanehashi S, Nagai K. Effects of various liquid organic solvents on solvent-induced crystallization of amorphous poly(lactic acid) film. Journal of Applied Polymer Science. 2012;129:1607-17.

[33] Yao J, Bastiaansen C, Peijs T. High Strength and High Modulus Electrospun Nanofibers. Fibers. 2014;2:158-86.

[34] Fu S-Y, Lauke B. Effects of fiber length and fiber orientation distributions on the tensile strength of short-fiber-reinforced polymers. Composites Science and Technology. 1996;56:1179-90.

[35] Garkhail S, Heijenrath R, Peijs T. Mechanical properties of natural-fibre-mat-reinforced thermoplastics based on flax fibres and polypropylene. Applied Composite Materials. 2000;7:351-72. 
[36] Zhang X, Nakagawa R, Chan KHK, Kotaki M. Mechanical property enhancement of polylactide nanofibers through optimization of molecular weight, electrospinning conditions, and stereocomplexation. Macromolecules. 2012;45:5494-500.

[37] Wu D, Wu L, Zhou W, Sun Y, Zhang M. Relations between the aspect ratio of carbon nanotubes and the formation of percolation networks in biodegradable polylactide/carbon nanotube composites. Journal of Polymer Science Part B: Polymer Physics. 2010;48:479-89.

[38] Toncheva A, Mincheva R, Kancheva M, Manolova N, Rashkov I, Dubois P, Markova N. Antibacterial PLA/PEG electrospun fibers: Comparative study between grafting and blending PEG. European Polymer Journal. 2016;75:223-33. 